\title{
Experimental and theoretical analysis of limit cycle bistability in a free-running VCSEL
}

\author{
Martin Virte ${ }^{a, b}$, Marc Sciamanna $^{b}$, Emeric Mercier $^{a, b}$ and Krassimir Panajotov ${ }^{a, c}$ \\ ${ }^{a}$ Brussels Photonics Team, Dept. of Applied Physics and Photonics (B-Phot TONA), Vrije \\ Universiteit Brussel, Pleinlaan 2, Brussels, Belgium. \\ ${ }^{b}$ OPTEL Research Group, Matèriaux optiques, photoniques et systèmes (LMOPS) EA-4423, \\ Supélec - Université de Lorraine, 2 rue Edouard Belin, F-57070 Metz, France. \\ ${ }^{c}$ Institute of Solid State Physics, 72 Tzarigradsko Chaussee Blvd., 1784 Sofia, Bulgaria.
}

\begin{abstract}
Laser diodes typically behave like damped oscillators: they are generally expected to only show damped relaxation oscillations toward a stable fixed point. In vertical-cavity surface-emitting lasers (VCSELs), the picture appears to be quite different as polarization dynamics can be experimentally observed including bifurcations to selfpulsation and even chaos. Physically, the circular geometry of VCSELs makes the polarization selection very weak and, thus, the additional degree of freedom can enable complex dynamical behavior in the laser diode.

Here we report on a new dynamical behavior in a free-running VCSEL: we observe a bistability between two limit cycles oscillating around two distinct elliptical polarization states whose main axes are symmetrical with respect to the polarization at threshold. Although the existence of two symmetric elliptical polarizations and the associated limit cycles are predicted by the San Miguel, Feng and Moloney (SFM) model, the hysteresis cycle observed experimentally highlights the importance of asymmetry in the dynamics from the elliptically polarized states. We demonstrate that this behavior can be accurately reproduced in theory within the SFM framework when taking into account a small misalignment between the phase and amplitude anisotropies of the laser cavity. Our results bring new light into VCSEL polarization dynamics and provide a very good qualitative agreement with the bifurcation scenario predicted by the SFM model.
\end{abstract}

Keywords: Vertical-Cavity Surface-Emitting Laser, Nonlinear Dynamics, Semiconductor Laser

\section{INTRODUCTION}

Compared to regular edge-emitting lasers, Vertical-Cavity Surface-Emitting Lasers (VCSELs) exhibit key advantages such as a circular beam, a significant threshold reduction and on-chip testing capability. Nevertheless many experimental studies during the past 20 years reported severe polarization instabilities including polarization switching, ${ }^{1-4}$ self-pulsating dynamics ${ }^{5-9}$ and polarization chaos $;{ }^{10}$ see Ref. ${ }^{11}$ for a recent review. Since those instabilities are major drawbacks for VCSEL applications, they motivated numerous studies and several mechanisms have been suggested to explain the polarization dynamics, including spin-relaxation processes, in the so-called spin-flip model or SFM. ${ }^{12,13}$ In the frame of these studies, elliptically polarized (EP) states have been reported experimentally, ${ }^{6}$ but there have been only few investigations of their underlying dynamics. ${ }^{14}$ Yet, it was recently demonstrated that these EP states play an essential role in the bifurcation scenario creating polarization chaos in a solitary VCSEL. ${ }^{7,8,10}$

According to the SFM model as described in Ref., ${ }^{13}$ the pitchfork bifurcation destabilizing the linear polarization stable at threshold creates two symmetrical EP states. In theory, the bifurcation scenario will then be strictly identical for both orientations: the ellipticity evolution will be the same - although with different orientations of the main axis, the two Hopf bifurcations destabilizing the EP states will appear at the same current

Further author information: (Send correspondence to M.V., M.S. or K.P.)

M.V.: E-mail: mvirte@b-phot.org, Telephone: +32 (0) 26293662

M.S.: E-mail: marc.sciamanna@supelec.fr, Telephone: +33 (0) 387764705

K.P.: E-mail: kpanajot@b-phot.org, Telephone: +32 (0) 26293567 
and will create two identical limit cycles, i.e. self-pulsating solutions, oscillating at the same frequency. Because of this equivalence, the laser should settle at random on one or the other EP state when the current is increased. Experimentally, however, we observe that the laser always selects the same orientation. Moreover, reproducible switchings between the two orientations have been reported, ${ }^{8}$ which confirm that the strict equivalence as seen in theory is not a viable option.

Here we unveil an even more surprising phenomenon: a bistability between two limit cycles oscillating around two EPs with main axes symmetrical with respect to the linear polarization at threshold. The two cycles exhibit different frequencies and amplitudes, and we unveil a clear hysteresis cycle when changing the injection current of the laser. Theoretically, we show that this behavior can be accurately reproduced by breaking the symmetry between the two EP states. In particular, when including a small misalignement between the phase and amplitude anisotropies considered in the SFM framework, we obtain a convincing agreement between experimental results and theoretical predictions.

This contribution is organized as follows: the experiment and experimental results are described in section 2 . In section 3, we present simulation results based on an extended SFM model, including a misalignment between the cavity anisotropies, which shows good qualitative agreement with the experimental findings. Finally, we discuss the results obtained and the perspectives for future work in section 4.

\section{EXPERIMENTAL RESULTS: OBSERVATION OF A BISTABILITY OF LIMIT CYCLES}

The VCSEL analyzed in this experiment is a single-mode InGaAs submonolayer quantum dot VCSEL emitting at $990 \mathrm{~nm}$ stabilized in temperature at $22^{\circ} \mathrm{C}$. The device has been described in details in Ref. ${ }^{15}$ The laser is collimated with an aspheric lens and coupled into a multimode fiber for measurement. Reflections from the fiber front facet are prevented by using an optical isolator and polarization measurement are performed by rotating a half-waveplate. In this section, we define the polarization at $0^{\circ}$ as the linear polarization at threshold. The output power is measured using a Newport Power Meter, and the time-series are recorded using a fastphotodetector (NewFocus 1554-B, 12GHz), an electronic amplifier (NewFocus 1422LF, 20GHz) and a large bandwidth oscilloscope (Agilent DS0X92504A, 25GHz, $80 \mathrm{GS} / \mathrm{s}$ ). The Radio-frequency spectra are obtained through a Fast Fourier Transform of the recorded time-series.
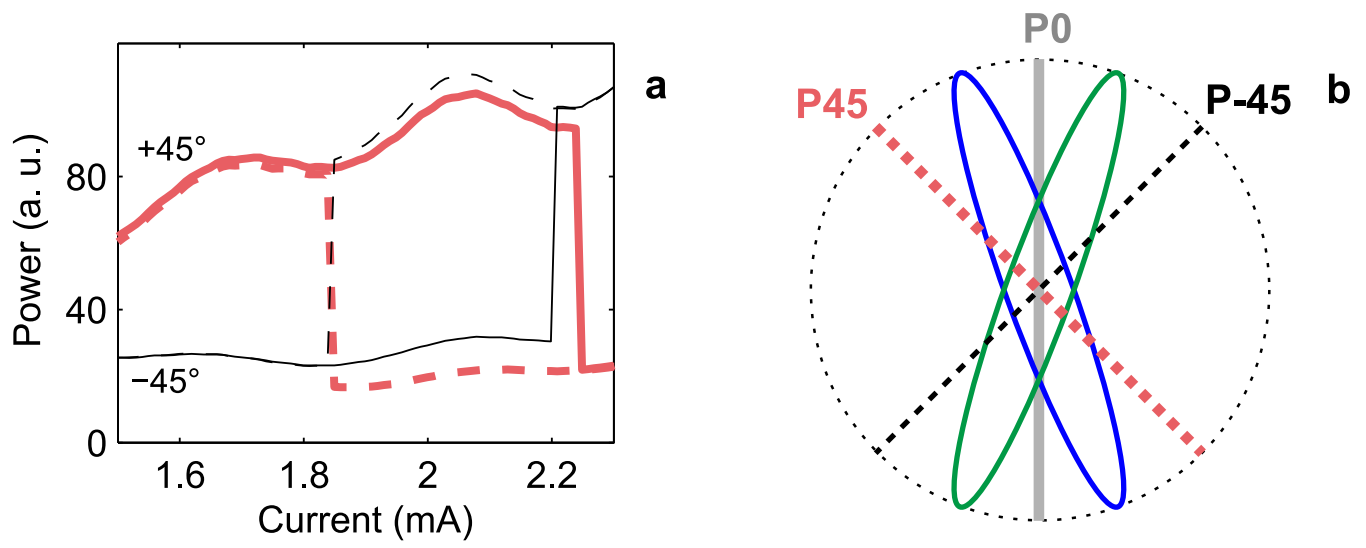

Figure 1. Experimental observations of the bistability. (a) Polarization LI curve at $+45^{\circ}$ (thick, red) and $-45^{\circ}$ (thin, black) for an increasing (solid) and a decreasing injection current (dashed). (b) Scheme of the different polarization states invoveld: linear polarization at threshold is the vertical gray line P0, and the two EP states around which the limit cycles oscillate are the two ellipses symmetrical with respect to P0. The dashed lines show the polarizations used for the measurement of the LI curve: $+45^{\circ}$ (thick red) and $-45^{\circ}$ (thin black).

At threshold the laser starts emitting on the vertical polarization P0, see Fig.1-(b), but an increase of the current destabilizes this steady-state and creates two symmetrical EP states, shown as two ellipses. To discriminate these two solutions, we need to observe the polarizations at $\pm 45^{\circ}$ where the contrast between the 
two EPs is maximized. This is what we do in fig.1-(a), where we plot the polarization resolved output power evolution for increasing (solid line) and decreasing injection current (dashed line). Thus we observe a clear switching between the two solutions - for current around $2.2 \mathrm{~mA}$, similar to what as been reported in Ref. ${ }^{8}$ When decreasing the current, we unveil an hysteresis cycle between the two EP states for currents between $1.8 \mathrm{~mA}$ and $2.2 \mathrm{~mA}$. However these two solutions are not stationary as can be seen from the time-series measurement given in fig.2. Based on the bifurcation diagrams generated from experimental time-traces, we observe two distinct limit cycles. The first one, the one stable at lower current, shows a larger amplitude and a frequency around $6.25 \mathrm{GHz}$. When the current is increased and reaches $1.95 \mathrm{~mA}$, we observe a sudden dynamical change, see Fig.2-(a, b), the amplitude of the oscillations decreases and the frequency goes up to $6.55 \mathrm{GHz}$. Then, when the current is decreased, the oscillations are strongly reduced and the main peak of the FFT spectrum vanishes, see Fig.2-(c, d). Under $1.77 \mathrm{~mA}$, the system finally settles back to the first limit cycle hence closing the hysteresis loop.
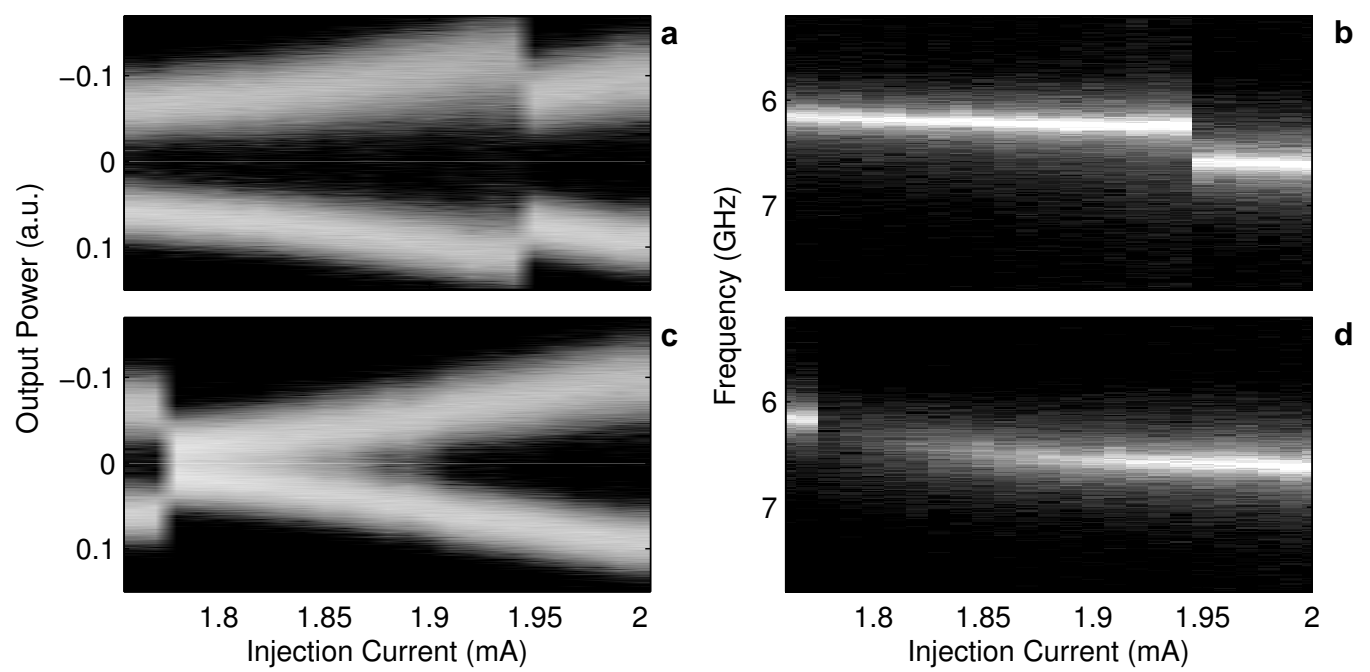

Figure 2. Identification of the limit cycles. (a, c) Plot of the time-series extrema versus increasing (top, a) and decreasing current (bottom, c). (b, d) FFT of the time-series versus increasing (top, b) and decreasing current (bottom, d). Time series considered for all panels are recorded at $0^{\circ}$, i.e. polarization at threshold, with DC removed.

Because the device is very sensitive to external conditions such as stress from the probes, the range of current at which the bistability appears can vary between different measurements. In particular, the hysteresis is observed at two different ranges of current between Fig. 1-(a) and Fig.2.

\section{SFM MODEL: ANISOTROPY MISALIGNMENT BREAKS EP STATE SYMMETRY}

As stated earlier, in the SFM model described in Ref., ${ }^{12,13}$ no distinction can be made between the two EP states, and the system is therefore expected to choose randomly between the two. On the other hand, we experimentally report on a biased situation where the two elliptical polarizations are not equivalent, as demonstrated by the hysteresis between the two limit cycles. Hence to model this new behavior we need to identify a mechanism that could break the symmetry between the EP states. We demonstrate here that a misalignment between the phase and amplitude anisotropy has such an effect. Based on this modified SFM model, we then show that a good agreement between theoretical prediction and experimental results can be achieved.

The SFM model considers the right and left circular polarizations as two different emission processes with separated carrier reservoirs; the coupling between them appears through the spin-flip relaxation processes that equilibrate carrier population. The cavity anisotropies then select the main orthogonal axes of polarization and introduce an asymmetry between the two, but the symmetry of the EP states remain. Yet, the introduction of 
a misalignment between the phase and amplitude anisotropies can break this symmetry. The impact of such misalignment on the steady-states of the SFM model has already been investigated previously, ${ }^{16,17}$ but no studies were conducted to observe the impact on the dynamical states of the laser. This modified SFM model then writes as follow:

$$
\begin{gathered}
\frac{d E_{+}}{d t}=\kappa \cdot(1+i \alpha) \cdot(N+n-1) \cdot E_{+}-\left(i \cdot \gamma_{p}+(\cos (2 \cdot \theta)-i \cdot \sin (2 \cdot \theta)) \cdot \gamma_{a}\right) \cdot E_{-} \\
\frac{d E_{-}}{d t}=\kappa \cdot(1+i \alpha) \cdot(N-n-1) \cdot E_{-}-\left(i \cdot \gamma_{p}+(\cos (2 \cdot \theta)+i \cdot \sin (2 \cdot \theta)) \cdot \gamma_{a}\right) \cdot E_{+} \\
\frac{d N}{d t}=\mu-N-(N+n) \cdot\left|E_{+}\right|^{2}-(N-n) \cdot\left|E_{-}\right|^{2} \\
\frac{d n}{d t}=-\gamma_{s} \cdot n-(N+n) \cdot\left|E_{+}\right|^{2}+(N-n) \cdot\left|E_{-}\right|^{2}
\end{gathered}
$$

With $E_{ \pm}$the field for the left $(+)$and right (-) circular polarization, $N$ the normalized total carrier population and $n$ the normalized carrier population difference between the two reservoirs. The field decay rate is $\kappa$, the spin-flip relaxation rate is $\gamma_{s}$, the injection current is $\mu$ and $\alpha$ is the linewidth enhancement factor. The cavity anisotropies are modeled by the phase anisotropy or birefringence $\gamma_{p}$, the amplitude anisotropy $\gamma_{a}$ and $\theta$, the angle between the phase and amplitude anisotropy axes - i.e. the axis of maximum frequency and the axis of maximum losses. All parameters and variables are dimensionless and the time is normalized by the carrier lifetime.

For numerical convenience, we reduce eqs. 1-4 it to a set of 5 real equations considering only the phase difference between the two electrical field for the left and right circular polarization similarly to what was done in Ref. ${ }^{18}$ To reproduce the behavior reported experimentally we use the following parameter values: $\kappa=600, \alpha=3$, $\gamma_{s}=100, \gamma_{p}=35, \gamma_{a}=-10$ and $\theta=0.023$.
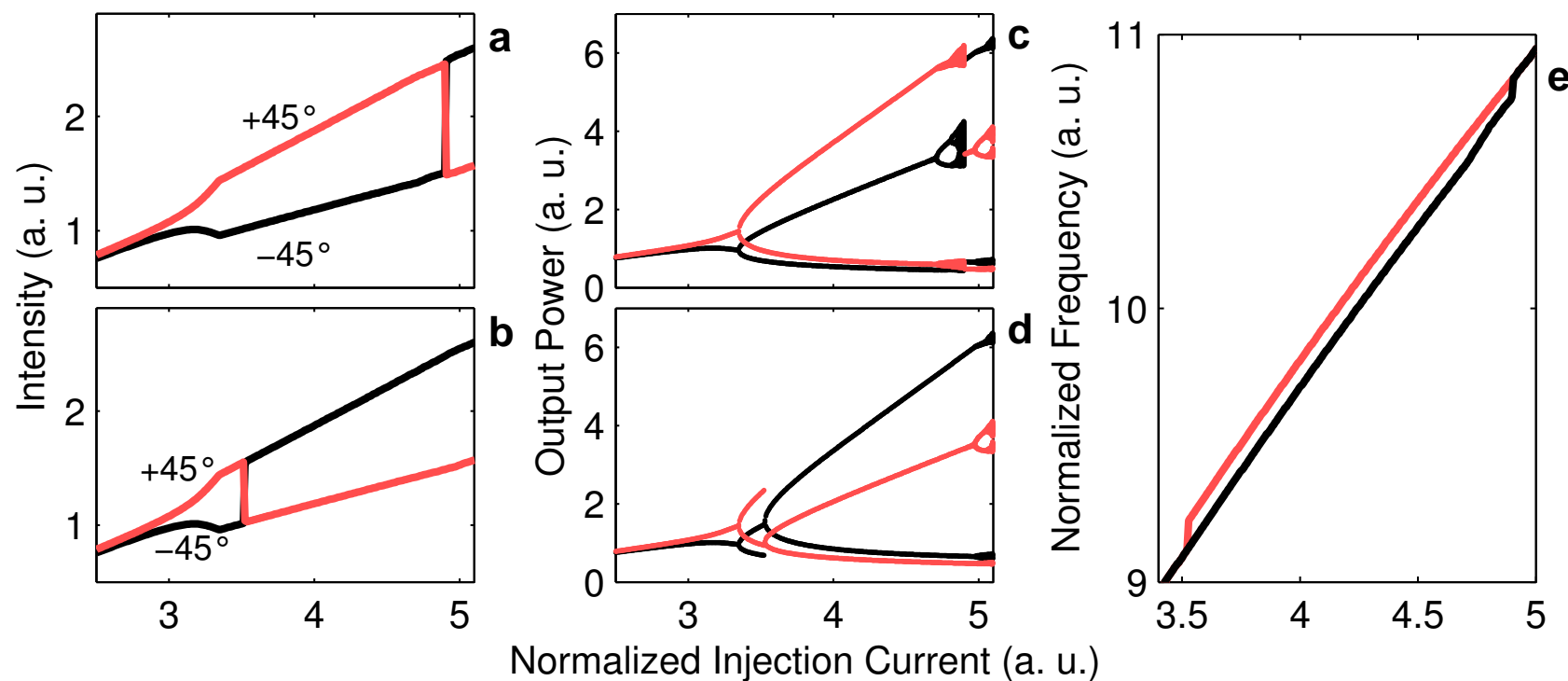

Figure 3. Simulated bifurcation diagram for increasing and decreasing injection current. (a, b) Mean output power for polarization at $\pm 45^{\circ}$ for increasing (a) and decreasing injection current (b). (c, d) Extrema of the polarization resolved time-series at $\pm 45^{\circ}$ in red $(+)$ and black (-) for increasing (c) and decreasing current (d). (e) Normalized frequency evolution for increasing (black) and decreasing (red) injection current.

As seen in fig.3-(a) and (c), at threshold the laser first emits linearly polarized light, but no pitchfork bifurcation appears to destabilize the state stable at threshold. Instead, we observe a smooth increase of the 
state ellipticity until a Hopf bifurcation occurs around $J \approx 3.3$ hence creating a self-pulsating solution. The newly created limit cycle oscillates around the now unstable EP state: we call this solution LC+. For increasing injection current, a sequence of bifurcation occurs at $J=4.8$ that destabilize LC+ and a new limit cycle (LC-) is unveiled. LC- exhibits almost perfectly reversed polarization properties compared to LC+, but, as can be seen from Fig.3-(e), its frequency is slightly different. We estimate the gap to be grossly around 0.1, i.e. $100 \mathrm{MHz}$ for a carrier lifetime of $1 \mathrm{~ns}$. From this point, if we decrease the current we unveil a relatively large bistability region between LC- and LC+. We observe that the amplitude of LC- oscillations decreases along with the current; the laser even reaches a steady-state for a tiny range of current. Then at $J=3.5$, the laser settles back to $\mathrm{LC}+$, hence closing the hysteresis cycle.

\section{CONCLUSION}

To sum up, we report a peculiar limit-cycle bistability in a free-running VCSEL where the two cycles oscillate around two different elliptically polarized states with main axes symmetrical with respect to the linear polarization at threshold. We also demonstrate that this behavior can be qualitatively well reproduced when considering a small misalignment between the phase and amplitude anisotropies in the laser cavity.

Our work therefore provides further evidence of the validity of the SFM model as a framework to describe the nonlinear dynamics and polarization properties of VCSELs. We also highlight a new dynamical behavior in a free-running VCSEL and bring new light on the role of the anisotropies in the selection and dynamics of VCSEL polarization. In addition, we motivate additional experimental and theoretical investigations for a better understanding and a better evaluation of the impact of these anisotropies.

\section{ACKNOWLEDGMENTS}

The authors acknowledge support from the Conseil Régional de Lorraine, Fondation Supélec, FWO-Vlaanderen, the METHUSALEM program of the Flemish government, and the interuniversity attraction poles program of the Belgian Science Policy Office (grant no. IAP P7-35 "Photonics@be").

\section{REFERENCES}

[1] Choquette, K., "Temperature dependence of gainguided verticalcavity surface emitting laser polarization," Appl. Phys. Lett. 64, 2062 (1994).

[2] Choquette, K. D., Schneider, R. P., Lear, K. L., and Leibenguth, R. E., "Gain-dependent polarization properties of vertical-cavity lasers," IEEE J. Sel. Top. Quant. Electron. 1(2), 661 (1995).

[3] van Exter, M., Willemsen, M., and Woerdman, J., "Polarization fluctuations in vertical-cavity semiconductor lasers," Phys. Rev. A 58, 4191-4205 (Nov. 1998).

[4] Panajotov, K., Ryvkin, B., Danckaert, J., Peeters, M., Thienpont, H., and Veretennicoff, I., "Polarization switching in VCSEL's due to thermal lensing," IEEE Photon. Technol. Lett. 10, 6-8 (Jan. 1998).

[5] Ackemann, T. and Sondermann, M., "Characteristics of polarization switching from the low to the high frequency mode in vertical-cavity surface-emitting lasers," Appl. Phys. Lett. 78(23), 3574 (2001).

[6] Sondermann, M., Ackemann, T., Balle, S., Mulet, J., and Panajotov, K., "Experimental and theoretical investigations on elliptically polarized dynamical transition states in the polarization switching of verticalcavity surface-emitting lasers," Opt. Commun. 235, 421-434 (May 2004).

[7] Olejniczak, L., Sciamanna, M., Thienpont, H., Panajotov, K., Mutig, A., Hopfer, F., and Bimberg, D., "Polarization switching in quantum-dot vertical-cavity surface-emitting lasers," IEEE Photon. Technol. Lett. 21(14), 1008-1010 (2009).

[8] Olejniczak, L., Panajotov, K., Thienpont, H., Sciamanna, M., Mutig, A., Hopfer, F., and Bimberg, D., "Polarization switching and polarization mode hopping in quantum dot vertical-cavity surface-emitting lasers.," Opt. Express 19, 2476-84 (Jan. 2011). 
[9] Virte, M., Panajotov, K., and Sciamanna, M., "Bifurcation to nonlinear polarization dynamics and chaos in vertical-cavity surface-emitting lasers," Phys. Rev. A 87, 013834 (Jan. 2013).

[10] Virte, M., Panajotov, K., Thienpont, H., and Sciamanna, M., "Deterministic polarization chaos from a laser diode," Nature Photon. 7, 60-65 (Nov. 2012).

[11] Panajotov, K. and Prati, F., "Polarization dynamics of vcsels," in [VCSELs], 181-231, Springer (2013).

[12] San Miguel, M., Feng, Q., and Moloney, J., "Light-polarization dynamics in surface-emitting semiconductor lasers," Phys. Rev. A 52(2), 1728-1739 (1995).

[13] Martin-Regalado, J., Prati, F., San Miguel, M., and Abraham, N., "Polarization properties of vertical-cavity surface-emitting lasers," IEEE J. Quant. Electron. 33, 765-783 (May 1997).

[14] Prati, F., Caccia, P., Bache, M., and Castelli, F., "Analysis of elliptically polarized states in vertical-cavitysurface-emitting lasers," Phys. Rev. A 69, 033810 (Mar. 2004).

[15] Hopfer, F., Mutig, A., Kuntz, M., Fiol, G., Bimberg, D., Ledentsov, N. N., Shchukin, V. a., Mikhrin, S. S., Livshits, D. L., Krestnikov, I. L., Kovsh, a. R., Zakharov, N. D., and Werner, P., "Single-mode submonolayer quantum-dot vertical-cavity surface-emitting lasers with high modulation bandwidth," Appl. Phys. Lett. 89(14), 141106 (2006).

[16] Travagnin, M., van Exter MP, Jansen van Doorn AK, and Woerdman, J., "Role of optical anisotropies in the polarization properties of surface-emitting semiconductor lasers.," Phys. Rev. A 54, 1647-1660 (Aug. 1996).

[17] Travagnin, M., "Linear anisotropies and polarization properties of vertical-cavity surface-emitting semiconductor lasers," Phys. Rev. A 56, 4094-4105 (Nov. 1997).

[18] Erneux, T., Danckaert, J., Panajotov, K., and Veretennicoff, I., "Two-variable reduction of the San MiguelFeng-Moloney model for vertical-cavity surface-emitting lasers," Phys. Rev. A 59, 4660-4667 (June 1999). 